Open Access

Original Article

\title{
Gastrointestinal complications in critically ill children: Experience from a resource-limited country
}

\author{
Sidra Ishaque ${ }^{1}$, Mariam Shakir2, \\ Asma Ladak ${ }^{3}$, Anwar Ul Haque ${ }^{4}$
}

\begin{abstract}
Objectives: To determine the frequency and predictors of outcome of gastrointestinal complications (GIC) in critically ill children.

Methods: This descriptive study was prospectively conducted in The Pediatric Intensive Care Unit (PICU), The Aga Khan University Hospital (AKUH), Karachi, from September 2015 to January 2017. After obtaining approval from the Ethical Review Committee of AKUH and informed consent from the parents, all children (aged one month to 18 years), of either gender, admitted to the Pediatric Intensive Care Unit (PICU) during the study period were included. The frequency of the defined GIC: vomiting, high gastric residue volume (GRV), diarrhea, constipation, and gastrointestinal bleed were recorded daily for the first week of the PICU stay. The data was collected by the primary investigator on a predesigned data collection form with inclusion of variables and predictors in light of existing literature and local expertise. The questionnaire was shared with the Pediatric Critical Care Medicine faculty and a consensus was sought on the elements to be incorporated.

Results: GIC developed within the first 48 hours of admission in 78 (41\%) patients. Of the patients who developed GIC, 37 (47.4\%) patients developed high GRV: 31 (39.7\%) patients developed constipation, 18 (23.1\%) patients developed vomiting, 14 (17.9\%) patients developed abdominal distension. With regards to prevalence by occurrence, $32 / 78$ (41\%) of patients presented with two Gl complications, followed by 21 patients $(27 \%)$ who presented with a single GIC. Only 11 patients $(14 \%)$ presented with more than three complications. Median length of stay was higher in patients with GIC (8 days) than with those who did not develop GIC (4 days). The frequency of gastrointestinal complications was significantly higher in children receiving mechanical ventilation, on sedatives and relaxants and those with multiorgan dysfunction syndrome (MODS) and inotropes

Conclusion: GI complications are a frequent occurrence in the PICU and are associated with worse clinical outcomes. The use of sedative drugs and the presence of shock with MODS were amongst the important contributing factors.
\end{abstract}

KEYWORDS: Gastrointestinal Complications, Critically Ill, Children.

doi: https://doi.org/10.12669/pjms.37.3.3493

How to cite this:

Ishaque S, Shakir M, Ladak A, Anwar-Ul-Haque. Gastrointestinal complications in critically ill children: Experience from a resource-limited country. Pak J Med Sci. 2021;37(3):657-662. doi: https://doi.org/10.12669/pjms.37.3.3493

This is an Open Access article distributed under the terms of the Creative Commons Attribution License (http://creativecommons.org/licenses/by/3.0), which permits unrestricted use, distribution, and reproduction in any medium, provided the original work is properly cited.

Correspondence:

Dr. Sidra Ishaque, FCPS.

Assistant Professor,

Pediatric Critical Care Medicine,

Department of Pediatrics and Child Health,

The Aga Khan University Hospital, Karachi, Pakistan.

Email: sidra.ishaque@aku.edu

* Received for Publication:

* $1^{\text {st }}$ Revision Received:

* $2^{\text {nd }}$ Revision Received:

* Final Revision Accepted:
August 17, 2020

August 22, 2020

January 16, 2021

January 27, 2021

\section{INTRODUCTION}

Gastrointestinal complications (GIC) are common in critically ill children. ${ }^{1}$ GIC are commonly observed, as either a primary reason for admission or as a part of multiple organ dysfunction syndrome (MODS) in children admitted in the Pediatric Intensive Care Unit (PICU). Despite its prominence in critically ill patients with MODS, 
GIC are not included in any of the scoring systems used to assess organ failure in critical illness. A critically ill patient may develop GIC throughout his illness, e.g., high gastric residual volumes (GRVs), constipation, or vomiting. GIC has also however has been associated with high morbidity in critically ill children. ${ }^{2}$

GIC are often ignored in PICU which often delays enteral nutrition preventing patients from getting adequate calorie and protein intake, ultimately leading to acquired malnutrition in these patients. The lack of a uniform standard definition of GIC adds to delays in its recognition. ${ }^{3,4}$ Critical illness can result in intestinal mucosal ischemia, that further damages the gut barrier function. ${ }^{5}$ Recently with the increasing awareness of GIC in critically ill patients, the Working Group on Abdominal Problems (WGAP) of the European Society of Intensive Care Medicine (ESICM) proposed a set of definitions of acute gastrointestinal injury (AGI) in critical illnesses in adults for both clinical and research purposes. ${ }^{2}$ However, there is no such definition available for the pediatric population. Also, the associations between AGI grade, the severity of GI dysfunction, and adverse outcome remains to be elucidated.

Reintam et al reported an incidence of GIC of $59 \%$ in their mixed-ICU population. ${ }^{5}$ Common GIC included vomiting, high gastric residual volume (GRV), bowel distension, diarrhea, and GI bleeding, in their report. There is limited published literature on the frequency and outcome of GIC related to enteral feeding in critically ill children. ${ }^{5,6}$ Critically ill patients with GIC have a prolonged length of stay in ICU and higher mortality as compared to those without gastrointestinal complications. , $, 5,6,7$

The epidemiological data on GIC in PICU is scarce. However, our experience with sick children has shown GIC to be associated with worse patient outcomes. We, therefore, aimed to determine the frequency of GIC and its association with outcomes at our PICU.

\section{METHODS}

After obtaining approval from the Institutional Ethical Review Committee (Ref \# 3548-PedERC-15, Dated May 20, 2020), this descriptive study was prospectively conducted in The Pediatric ICU of The Aga Khan University Hospital, Karachi from September 2015 to January 2017. Ours is a multi-disciplinary eight bedded
PICU, with three trained intensivists providing 24-hour coverage.

Based on a study by Lopez-Herce et al, that reported a frequency of GIC in critically ill children to be $11.5 \%{ }^{3}$ with a margin of error of $5 \%$, a power $(1-\beta)$ of $80 \%, 190$ patients were included in this study by using nonprobability, consecutive sampling technique. We routinely start enteral feeding through a nasogastric tube within 4-8 hours after admission/resuscitation unless contraindicated for enteral feeding or nasogastric tube (NGT). The continuous feeding is preferred in patients who are receiving vasoactive drugs.

A nutrition protocol (see annex) was used to initiate and monitor feeding in our patients. GRV was considered to be high when it exceeded half the volume of the previous feed given. GRV is assessed every four hours after ICU admission for a total of six times per day. Children admitted primarily with gastrointestinal symptoms like GI Bleeding or Acute Pancreatitis, Status-Post laparotomy, and those who stayed less than 24 hours in the PICU were excluded. Gastrointestinal complications were defined as the presence of at least one of the following pre-defined gastrointestinal problems in patients during their first-week of PICU stay as shown in Table-I. ${ }^{3}$ All GIC were recorded, including the number of episodes and day of presentation.

The primary endpoint was defined as the frequency of gastrointestinal complications developed in critically ill children during the first-week in PICU. Secondary outcomes included the association of GIC to mortality, malnutrition, length of PICU stay, and the risk factors for the development of GIC. A structured data collection form was used for data collection, including demographic data, clinical variables, including admitting diagnosis, PRISM-III score for severity assessment, ${ }^{8}$ use of PICU therapies like mechanical ventilation and inotropes, presence of MODS according to IPSCC $2005^{7}$ as well as predefined GICs (as shown in Table-I) and hospital discharge as alive or deceased.

All data were entered and analyzed using Statistical packages for social science version 22 (SPSS Inc., Chicago, IL). The quantitative variables like age, weight, height, length of stay, and duration of mechanical ventilation are expressed as mean with standard deviation and qualitative variables are expressed as percentages or range. T-test and chi-square tests were used for 
continuous and categorical variables respectively. Binary logistic regression analysis was performed for the assessment of factors associated with the development of GI complications. P-value $\leq 0.05$ was considered as significant.

\section{RESULTS}

From a total of 448 patients screened for eligibility, 190 patients met inclusion criteria and $78(41 \%)$ developed one or more GIC during the first week of their PICU admission. The GIC have been defined in Table-I. The median age of the study population was 43 months (range $1 \mathrm{~m}$-18years, interquartile range $=78$ ). Our study population consisted of 101 (53.5\%) males and 89 $(56.8 \%)$ females with a weight mean of weight of $10.2 \mathrm{~kg}$ (range $3.6-68 \mathrm{~kg}$ )

The overall median PRISM score of the study population was 6 [IQR (4-10)]. 78 patients $(41 \%)$ developed GIC during the study period. Of those, $27(34.6 \%)$ were admitted for CNS disorders, $19(24.4 \%)$ were admitted for miscellaneous disorders, 14 (17.9\%) for cardiovascular disorders (Congenital heart disease, repaired or unrepaired, cardiomyopathies, myocarditis), followed by $7.6 \%$ of children with a respiratory illness. Only $4(5.13 \%)$ were surgical patients. $36 / 78(46.1 \%)$ with MODS developed GIC while 68/78 (87.1\%) with GIC were mechanically ventilated. The length of stay was higher in patients with GIC (8 days) than with those who did not develop GIC (4 days). Patients who developed GIC had a median PRISM-III score of 8 . Among ICU therapies, 77\% of patients in the GIC group and $66 \%$ of patients in the non-GIC group required vasoactive inotropic support $(p=0.003)$. Sedatives including nalbuphine, and/or dexmedetomidine and neuromuscular blocking agents like atracurium and cisatracurium were prescribed in $68 \%$ of

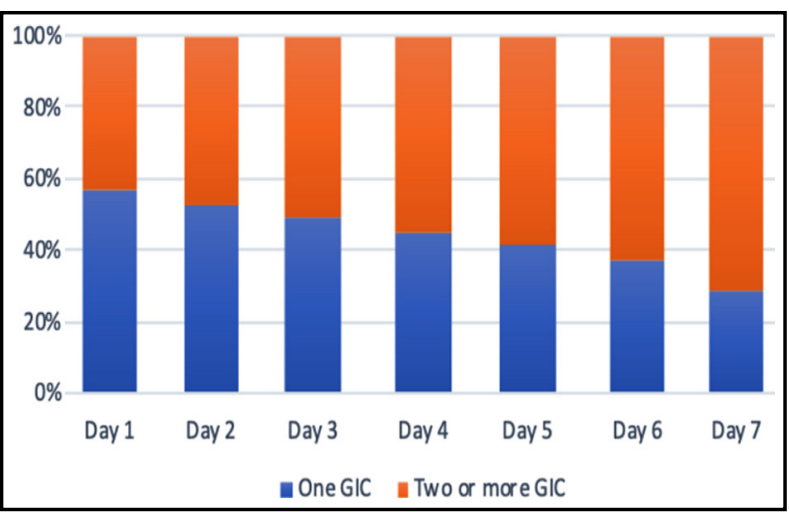

Fig.1: The prevalence of gastrointestinal (GI) symptoms daily during the first week in intensive care unit (ICU).

the patients with non-GIC patients requiring it more that the GIC group. Of the patients who expired, none were due to GIC; However, shock with MODS was the major underlying diagnosis (Table-II).

GIC developed within the first 48 hours of admission in $78(41 \%)$ patients. Of the patients who developed GIC, 37 (47.4\%) patients developed high GRV: 31 (39.7\%) patients developed constipation, $18(23.1 \%)$ patients developed vomiting, 14 (17.9\%) patients developed abdominal distension. With regards to prevalence by occurrence, 32/78 (41\%) of patients presented with two GI complications, followed by 21 patients $(27 \%)$ who presented with a single GIC. Only 11 patients (14\%) presented with more than three complications. The Prevalence of daily gastrointestinal symptoms during the first week in PICU (Fig.1).

On multivariate logistic regression analysis, the odds of having GI complications was 2.34 times higher in children receiving sedatives like nalbuphine, and/or dexmedetomidine during PICU stay. Both sedative and or relaxant use (p-value -0.010) and shock with MODS (p-value

Table-I: Definition of Gastrointestinal Complications (GIC).

Vomiting

High Gastric Residue

Diarrhea

Constipation

GI bleeding

Abdominal Distension
Involuntary, forceful expulsion of the contents of one's stomach

More than $50 \%$ of feed aspirated in previous 4 hour OR more than two-hour volume aspirated of the continuous feed being administered

More than 4 stools per day or change in the consistency of stool

No stool for more than 48 hours

Presence of macroscopic blood in vomitus, nasogastric aspirates or stool

Visibly distended abdomen and/OR Increase in abdominal girth by $2 \mathrm{~cm}$ from the baseline

OR X-ray abdomen shows significantly dilated bowel loops 
Sidra Ishaque et al.

Table-II: Demographic and Clinical Profile of Patients.

\begin{tabular}{|c|c|c|c|c|}
\hline Variables & All $(n=190)(\%)$ & $\operatorname{GICP}^{*}(n=78)(\%)$ & $\operatorname{GICN}^{\#}(n=112)(\%)$ & p-value \\
\hline Age in months (median) IQ range & $43(39-82)$ & $62(96)$ & $35(78)$ & 0.11 \\
\hline \multicolumn{5}{|l|}{ Gender } \\
\hline Male & $101(53.2)$ & $43(55.2)$ & $58(51.8)$ & \multirow{2}{*}{0.64} \\
\hline Female & $89(46.8)$ & $35(44.8)$ & $54(48.2)$ & \\
\hline PRISM-III score, Median (IQR) & $6(4-10)$ & $8(4-12)$ & $5(3-7)$ & 0.002 \\
\hline \multicolumn{5}{|l|}{ Diagnostic Category: } \\
\hline Central Nervous System & $52(27)$ & $27(34.6)$ & $25(22.3)$ & \\
\hline Respiratory System & $39(21)$ & $06(7.69)$ & $33(29.2)$ & \\
\hline Cardiovascular System & $26(14)$ & $14(17.9)$ & $12(10.7)$ & \multirow{4}{*}{0.41} \\
\hline Surgery & $16(8.4)$ & $04(5.13)$ & $12(10.7)$ & \\
\hline Trauma & $12(6.3)$ & $08(10.3)$ & $04(3.9)$ & \\
\hline Miscellaneous ${ }^{\circledR}$ & $45(23.3)$ & $19(24.4)$ & $26(23.2)$ & \\
\hline Co-morbidity & $40(21.1)$ & $18(23.0)$ & $22(19.6)$ & 0.57 \\
\hline Use of Mechanical Ventilation & $139(73.1)$ & $68(87.1)$ & $71(63.3)$ & $<0.001$ \\
\hline Shock including use of inotropes & $133(70)$ & $59(77.7)$ & $74(66.6)$ & 0.087 \\
\hline Use of sedatives & $130(68.4)$ & $63(80.76)$ & $67(59.8)$ & 0.002 \\
\hline Use of paralytics & $128(67.3)$ & $60(76.9)$ & $67(59.8)$ & 0.014 \\
\hline Outcome (deceased) & $10(5.3)$ & 07 (8.97) & $03(2.67)$ & 0.056 \\
\hline Length of stay (Day) Median (IQR) & $12(8-16)$ & $8(6-10)$ & $4(3-8)$ & $<0.001$ \\
\hline
\end{tabular}

*Gastrointestinal Complications, "No gastrointestinal complications, ^Standard deviation,

\$Multiorgan Dysfunction syndrome,

${ }^{\circledR}$ Sepsis/Hematology/Oncology/Near drowning/Poisoning/DKA/Tetanus/Infections/Autoimmune.

-0.002) have a statistically significant association with GIC (Table-III).

\section{DISCUSSION}

We found that $41 \%$ of our critically ill children had GIC during the first week of their PICU stay which is higher than that shown by Reintem $(16 \%)$ in their adult ICU patients. ${ }^{6}$ Adult colleagues have reported the frequency of GIC up to $60 \%{ }^{2,6,9}$ Our results are much higher than what has been reported in the literature; an incidence of $11.5 \% .^{3}$ The reason for such variability can be attributed to the lack of existing diagnostic criteria for GIC in both the adult and pediatric populations. Also, the fact that limited literature existing on gastrointestinal complications in critically ill children has been reported in specific populations, e.g., by Martinez et $\mathrm{al}^{10}$ [mechanically ventilated children] and another study from Spain $^{3}$ in children on transpyloric nutrition. Another possible reason for the higher frequency of GIC in our population could be the inclusion of all children, regardless of the type of feeding or ventilation status.

Twenty seven $(34.6 \%)$ of our patients with GIC had an underlying CNS condition. This is consistent with studies, ${ }^{3}$ where neurological

Table-III: Multivariable logistic regression analysis of factors determining the occurrence of GI complications.

\begin{tabular}{lccc}
\hline Variable & $\begin{array}{c}\text { Exp of } \beta \\
\text { (Prevalence } \\
\text { ratio) }\end{array}$ & $95 \%$ CI & p-value \\
\hline Use of Sedatives & 2.34 & $1.22,4.50$ & 0.001 \\
Shock with MODS & 0.48 & $0.22,0.70$ & 0.002 \\
\hline
\end{tabular}


conditions have been attributed as the three most commonly occurring diagnoses., ${ }^{3,10}$ Gastrointestinal dysfunction is amongst the common physiologic complications associated with traumatic brain injury. ${ }^{10}$ This feeding intolerance in particular may present with vomiting and abdominal distension. ${ }^{11}$ Mechanisms proposed to include impaired gastrointestinal motility, gastroesophageal reflux, delayed gastric emptying that may be related to reduced lower esophageal sphincter tone. ${ }^{12,13}$

We saw a high percentage of $37 / 78(47 \%)$ of patients with high gastric residual volumes (HGRVs) in our cohort. Similar results have previously been reported in literature. ${ }^{9,14}$ A randomized controlled trial by Horn et al from Brisbane defines a high GRV greater than $5 \mathrm{ml} / \mathrm{kg}$ in a group of critically ill pediatric patients. ${ }^{15} \mathrm{High}$ GRVs have been associated with interruptions in enteral feeding, thereby contributing to undernutrition in the PICU. ${ }^{10}$ In fact, on a similar note, the routine measurement of GRVs has been questioned as a tool to assess feeding intolerance. Although GRVs were reported to be the most frequently observed $(47.4 \%)$ complication in our cohort, it is imperative to define absolute criteria with regards to enteral feeding in critically ill children, and not solely base decision on one parameter. Interestingly, an observational study from Europe $^{16}$ states that not routinely measuring gastric residual volume as opposed to a routine measure of GRVs did not increase the incidence of ventilator acquired pneumonia, aspiration, or vomiting. These results offer food for thought and therefore we recommend the use of a standard feeding protocol to guide practice. Larger multicenter trials are required to provide clinicians with further evidence.

We found a significant association of the development of GIC with the use of sedative drugs like nalbuphine. Sedatives and muscle relaxants have been shown to affect enteral nutrition as they reduce gastrointestinal motility. ${ }^{17}$ Lopez-Herce et al have also mentioned sedatives and relaxants as an important reason for gastrointestinal morbidity in children. ${ }^{18}$ Our results show that enteral nutrition (EN) can be used in critically ill children despite being on sedatives and muscle relaxants, although constipation and abdominal distention must be carefully monitored in children especially those receiving continuous infusions of sedatives and relaxants.
In our study, shock was one of the most important risk factors for gastrointestinal complications. Enteral nutrition has been shown to increase splanchnic metabolic demands, which increase manifolds in states of sepsis and shock and may lead to altered tolerance and gastrointestinal complications. ${ }^{14}$ It has been advised to advance enteral nutrition with caution in critically ill children.

The critically ill children receiving mechanical ventilation were at high risk for developing GIC in our PICU. Mutlu et al narrated the various spectrums of GIC in critically ill patients receiving mechanical ventilation. ${ }^{14}$ The integration of GIC with MODS in critically ill children perpetuates mortality in our cohort and Reintam et al had similar observation. ${ }^{5.6}$

The benefits of a protocolized or guidelinedriven nutrition delivery strategy have been described in previous studies. ${ }^{19-21}$ Our current study reinforces the role of uniform guidelines in improving bedside nutrient delivery during critical illness. The use of EN algorithms/ protocols has been associated with decreased time to initiation of EN, increased EN delivery and decreased reliance on $\mathrm{PN}$, and increased likelihood of achieving nutrient delivery goals.

\section{CONCLUSIONS}

GI complications are a frequent occurrence in the PICU and are associated with worse clinical outcomes. High gastric residual volume was the most common GIC. The use of sedative drugs and presence of shock with MODS were important factors for GIC. We recommend multi-center studies and algorithmic protocol-driven approach to enteral nutrition for critically ill children.

Acknowledgements: The authors would like to acknowledge the efforts of Dr. Muhammad Khalid Saleem and Saad Nasir as "clinical investigators" and for their valuable input in terms of formatting and data analysis.

Grant Support E Financial Disclosures: None.

\section{REFERENCES}

1. Watson RS, Crow SS, Hartman ME, Lacroix J, Odetola FO. Epidemiology and Outcomes of Pediatric Multiple Organ Dysfunction Syndrome. Pediatr Crit Care Med. 2017;18(3_Suppl Suppl 1):S4-S16. 
2. Reintam Blaser A, Malbrain ML, Starkopf J, Fruhwald S, Jakob SM, De Waele J, et al. Gastrointestinal function in intensive care patients: terminology, definitions and management. Recommendations of the ESICM Working Group on Abdominal Problems. Intensive Care Med. 2012;38(3):384-394. doi: 10.1007/s00134-011-2459-y

3. Lopez-Herce J, Santiago MJ, Sanchez C, Mencia S, Carrillo A, Vigil D. Risk factors for gastrointestinal complications in critically ill children with transpyloric enteral nutrition. Eur J Clin Nutr. 2008;62(3):395-400. doi: 10.1038/ sj.ejcn. 1602710

4. Atasever AG, Ozcan PE, Kasali K, Abdullah T, Orhun G, Senturk E. The frequency, risk factors, and complications of gastrointestinal dysfunction during enteral nutrition in critically ill patients. Ther Clin Risk Manag. 2018;14:385391. doi: 10.2147/TCRM.S158492

5. Reintam A, Parm P, Redlich U, Tooding LM, Starkopf J, Kohler F, et al. Gastrointestinal failure in intensive care: a retrospective clinical study in three different intensive care units in Germany and Estonia. BMC Gastroenterol. 2006;6:19. doi: 10.1186/1471-230X-6-19

6. Reintam A, Parm P, Kitus R, Kern H, Starkopf J. Gastrointestinal symptoms in intensive care patients. Acta Anaesthesiol Scand. 2009;53(3):318-324. doi: 10.1111/ j.1399-6576.2008.01860

7. Goldstein B, Giroir B, Randolph A; International Consensus Conference on Pediatric Sepsis. International pediatric sepsis consensus conference: definitions for sepsis and organ dysfunction in pediatrics. Pediatr Crit Care Med. 2005;6(1):2-8. doi: 10.1097/01.PCC.0000149131.72248.E6

8. Pollack MM, Patel KM, Ruttimann UE. PRISM III: An update Pediatric Risk of Mortality score. Crit Care Med. 1996;24:743752. doi: 10.1097/00003246-199605000-00004

9. Montejo JC. Enteral nutrition-related gastrointestinal complications in critically ill patients: a multicenter study. The Nutritional and Metabolic Working Group of the Spanish Society of Intensive Care Medicine and Coronary Units. Crit Care Med. 1999;27(8):1447-1453. doi: 10.1097/00003246-199908000-00006

10. Martinez EE, Douglas K, Nurko S, Mehta NM. Gastric Dysmotility in Critically Ill Children: Pathophysiology, Diagnosis, and Management. Pediatr Crit Care Med. 2015;16(9):828-836. doi: 10.1097/PCC.0000000000000493

11. Krakau K, Omne-Ponten M, Karlsson T, Borg J. Metabolism and nutrition in patients with moderate and severe traumatic brain injury: A systematic review. Brain Inj. 2006;20:345-367. doi: 10.1080/02699050500487571

12. Tan M, Zhu JC, Yin HH. Enteral nutrition in patients with severe traumatic brain injury: Reasons for intolerance and medical management. Br J Neurosurg. 2011;25:2-8. doi: 10.3109/02688697.2010.522745
13. Kao $\mathrm{CH}$, ChangLai SP, Chieng PU, Yen TC. Gastric emptying in head-injured patients. Am J Gastroenterol. 1998;93:p1108-1112. doi: 10.1111/j.15720241.1998.00338.x

14. Mutlu GM, Mutlu EA, Factor P. GI complications in patients receiving mechanical ventilation. Chest. 2001;119(4):1222-1241.

15. Horn D, Chaboyer W, Schluter PJ. Gastric residual volumes in critically ill paediatric patients: a comparison of feeding regimens. Aust Crit Care. 2004;17(3):98-103. doi: 10.1016/ s1036-7314(04)80011-0

16. Tume LN, Bickerdike A, Latten L, Davies S, Lefèvre $\mathrm{MH}$, Nicolas GW, et al. Routine gastric residual volume measurement and energy target achievement in the PICU: A comparison study. Eur J Pediatr. 2017;176(12):1637-1644.

17. Mentec H, Dupont H, Bocchetti M, Cani P, Ponche F, Bleichner G. Upper digestive intolerance during enteral nutrition in critically ill patients: frequency, risk factors, and complications. Crit Care Med. 2001;29(10):1955-61. doi:10.1097/00003246-200110000-00018

18. López-Herce J. Gastrointestinal complications in critically ill patients: what differs between adults and children? Curr Opin Clin Nutr Metabol Care. 2009;12(2):180-185. doi: 10.1097/MCO.0b013e3283218285

19. Hamilton S, McAleer DM, Ariagno K, Barrett M, Stenquist $\mathrm{N}$, Duggan $\mathrm{CP}$, et al. A stepwise enteral nutrition algorithm for critically ill children helps achieve nutrient delivery goals. Pediatr Crit Care Med. 2014;15(7):583-589. doi: 10.1097/ pcc.0000000000000179

20. Petrillo-Albarano T, Pettignano R, Asfaw M, Easley K. Use of a feeding protocol to improve nutritional support through early, aggressive, enteral nutrition in the pediatric intensive care unit. Pediatr Crit Care Med. 2006;7(4):340344. doi: 10.1097/01.PCC.0000225371.10446.8F

21. Yoshimura S, Miyazu M, Yoshizawa S, So M, Kusama $\mathrm{N}$, Hirate $\mathrm{H}$, et al. Efficacy of an enteral feeding protocol for providing nutritional support after paediatric cardiac surgery. Anaesth Intens Care. 2015;43(5):587-593. doi: 10.1177/0310057X1504300506

\section{Authors Contribution:}

SI \& MS: Conceived, designed and did statistical analysis \& editing of manuscript, is responsible for integrity of the study.

AL \& AHQ: Reviewed and approved the final manuscript and helped with manuscript writing.
Authors:

1. Dr. Sidra Ishaque, FCPS. Department of Pediatrics and Child Health,

2. Dr. Mariam Shakir, FCPS. Department of Pediatrics and Child Health,

3. Asma Ladak, MBBS Medical College,

4. Dr. Anwar UI Haque MD. Department of Pediatrics, Liaquat National Hospital, Karachi, Pakistan.

1-3: The Aga Khan University Hospital, Karachi, Pakistan. 